\title{
A Longitudinal Study of the Long-Term Consequences of Drinking during Pregnancy: Heavy In Utero Alcohol Exposure Disrupts the Normal Processes of Brain Development
}

\author{
Catherine Lebel, ${ }^{1}$ Sarah N. Mattson, ${ }^{3}$ Edward P. Riley, ${ }^{3}$ Kenneth L. Jones, ${ }^{4}$ Colleen M. Adnams, ${ }^{5}$ Philip A. May, ${ }^{6}$ \\ Susan Y. Bookheimer, ${ }^{7}$ Mary J. 0'Connor, ${ }^{7}$ Katherine L. Narr, ${ }^{1}$ Eric Kan, ${ }^{2}$ Zvart Abaryan, ${ }^{2}$ and Elizabeth R. Sowell 1,2 \\ ${ }^{1}$ Department of Neurology, University of California at Los Angeles, Los Angeles, California 90095-1769, ${ }^{2}$ University of Southern California Keck School of \\ Medicine, Department of Pediatrics, Children's Hospital of Los Angeles, Los Angeles, California 90027, ${ }^{3}$ Department of Psychology, San Diego State \\ University, San Diego, California 92182-4611, ${ }^{4}$ Department of Pediatrics, University of California at San Diego, La Jolla, California 92123-5109, ${ }^{5}$ Department \\ of Psychiatry and Mental Health, University of Cape Town, Cape Town, South Africa, ${ }^{6}$ Department of Nutrition, Gillings School of Global Public Health, \\ Nutrition Research Institute, University of North Carolina, Chapel Hill, North Carolina 27599-7461, and 'Department of Psychiatry and Biobehavioral \\ Sciences, University of California at Los Angeles, Los Angeles, California 90095
}

Exposure to alcohol in utero can cause birth defects, including face and brain abnormalities, and is the most common preventable cause of intellectual disabilities. Here we use structural magnetic resonance imaging to measure cortical volume change longitudinally in a cohort of human children and youth with prenatal alcohol exposure (PAE) and a group of unexposed control subjects, demonstrating that the normal processes of brain maturation are disrupted in individuals whose mothers drank heavily during pregnancy. Trajectories of cortical volume change within children and youth with PAE differed from those of unexposed control subjects in posterior brain regions, particularly in the parietal cortex. In these areas, control children appear to show a particularly plastic cortex with a prolonged pattern of cortical volume increases followed by equally vigorous volume loss during adolescence, while the alcohol-exposed participants showed primarily volume loss, demonstrating decreased plasticity. Furthermore, smaller volume changes between scans were associated with lower intelligence and worse facial morphology in both groups, and were related to the amount of PAE during each trimester of pregnancy in the exposed group. This demonstrates that measures of IQ and facial dysmorphology predict, to some degree, the structural brain development that occurs in subsequent years. These results are encouraging in that interventions aimed at altering "experience" over time may improve brain trajectories in individuals with heavy PAE and possibly other neurodevelopmental disorders.

\section{Introduction}

Brain development, a dynamic process of progressive and regressive changes influenced by complex genetics and experience-dependent plasticity, may be altered in children with neurodevelopmental disorders such as prenatal alcohol

Received March 8, 2012; revised Aug. 7, 2012; accepted Aug. 14, 2012.

Author contributions: C.L., S.N.M., E.P.R., K.L.J., C.M.A., P.A.M., M.J.O., K.L.N., and E.R.S. designed research; C.L., S.N.M., E.P.R., K.L.J., C.M.A., P.A.M., M.J.O., K.L.N., and E.R.S. performed research; C.L., E.K., Z.A., and E.R.S. analyzed data; C.L., S.Y.B., and E.R.S. wrote the paper.

This work was performed in conjunction with the Collaborative Initiative on Fetal Alcohol Spectrum Disorders (www.cifasd.org), which is funded by grants from the National Institute on Alcohol and Alcohol Abuse (NIAAA). This work was also supported by NIAAA Grants U01 AA017122-01, to E.R.S., U24AA014811 to E.P.R., U01 AA014834 to S.N.M., and U01 AA11685 and R01 AA15134 to P.A.M.; National Institute of Drug Abuse Grant R01DA017831 to E.R.S.; National Institute of Child Health and Human Development Grant R01 HD053893 to E.R.S.; and March of Dimes Grant 6-FY2008-50 to E.R.S. C.L. receives salary funding from the Canadian Institutes for Health Research.

Correspondence should be addressed to Dr. Elizabeth R. Sowell, Director Developmental Cognitive Neuroimaging Laboratory, Professor of Pediatrics, Keck School of Medicine, University of Southern California, Division of Research on Children, Youth and Families, Department of Pediatrics, Children's Hospital Los Angeles, 4650 Sunset Boulevard, Mailstop \#130, Los Angeles, CA 90027. E-mail: esowell@chla.usc.edu.

DOI:10.1523/JNEUROSCI.1161-12.2012

Copyright $\odot 2012$ the authors $\quad 0270-6474 / 12 / 3215243-09 \$ 15.00 / 0$ exposure (PAE). Concomitant reductions in synaptic density (Huttenlocher, 1979; Huttenlocher and de Courten, 1987) and increases in axonal myelination (Yakovlev and Lecours, 1967) are hallmarks of experience-based neural plasticity and are consistent with selective specialization. It is thought that increasing efficiency, via myelination and synaptic pruning, occurs at the expense of extended plasticity, as underused connections are eliminated and are no longer available for use. While the study of maturation at the cellular level is limited to animal and postmortem human studies, in vivo magnetic resonance imaging (MRI) studies demonstrate similar patterns. Cortical volume and thickness increase initially, peak during late childhood or adolescence, and decline thereafter (Giedd and Rapoport, 2010). These changes vary regionally and, in general, parallel cognitive maturation.

The shape of developmental trajectories may be a better indicator of atypical neurodevelopment than cortical differences at any single time point (Shaw et al., 2006; Giedd et al., 2008), and these shapes have demonstrated differences in children and youth with attention deficit hyperactivity disorder (Shaw et al., 
2007), in children with child-onset schizophrenia (Thompson et al., 2001; Vidal et al., 2006), and even in healthy children with differing levels of intelligence (Shaw et al., 2006). Thus, studying maturational trajectories in neurodevelopmental disorders may lend new insight into these disorders and potential treatment strategies, which could vary depending on the cognitive and behavioral deficits and the age of the child.

Children and adolescents with PAE often exhibit facial dysmorphology, growth restriction, and a variety of cognitive, behavioral, and/or neurological problems (Riley et al., 2011), including cortical gray matter abnormalities (Archibald et al., 2001; Sowell et al., 2008a; Zhou et al., 2011; Chen et al., 2012; Yang et al., 2012a). However, little is known about longitudinal brain development in this population. Two cross-sectional studies examining subcortical gray matter volumes (Nardelli et al., 2011) and cortical thickness (Zhou et al., 2011) found no significant differences in linear development trajectories between alcohol-exposed subjects and controls, although qualitative observations suggest group differences. Longitudinal studies have more power to detect change and may reveal abnormalities not apparent in cross-sectional studies.

The purpose of this study was to examine trajectories of cortical change over time in children with and without PAE, and to test relationships between cortical development and cognitive/ behavioral function. Given that higher intelligence is associated with later peaks of cortical thickness (Shaw et al., 2006), we hypothesized that children and adolescents with PAE would reach peak cortical volume earlier than their unexposed peers, and that individuals with greater facial dysmorphology and intellectual deficits would show more aberrant cortical maturation during the interscan interval.

\section{Materials and Methods}

Subjects. This study was part of the Collaborative Initiative on Fetal Alcohol Spectrum Disorders (CIFASD) and included data from 133 subjects studied at three sites: Los Angeles, California $(n=36: 17$ male/19 female, 13 control/23 alcohol-exposed, 33 right-handed/3 left-handed); San Diego, California ( $n=18: 12$ male/6 female, 10 control/ 8 alcoholexposed, 15 right-handed/3 left-handed); and Cape Town, South Africa $(n=79: 45$ male/34 female, 40 control/39 alcohol-exposed, 76 righthanded/3 left-handed). Subjects were aged 5.7-15.9 years (12.4 \pm 2.6 years) at the time of their first MRI scan, and were all scanned again $\sim 2$ years later (mean time gap: $1.8 \pm 0.5$ years) at the same site with the same imaging protocol. Four subjects in Los Angeles received a third scan (mean age gap between second and third scans, $2.0 \pm 0.2$ years).

Control subjects identified their race as American Indian/Alaska native $(n=3)$, Asian $(n=4)$, African American $(n=3)$, Caucasian $(n=$ $10)$, Cape Colored $(n=40)$, or mixed race $(n=1)$. Alcohol-exposed subjects identified themselves as Asian $(n=1)$, African American $(n=$ $5)$, Caucasian $(n=25)$, or Cape Colored $(n=39)$. Five controls and 10 alcohol-exposed subjects were Hispanic/Latino. Fifty-six controls and 56 alcohol-exposed subjects were not Hispanic/Latino. Two controls and four alcohol-exposed subjects did not identify their race.

Selection criteria excluded control subjects with exposure in excess of 1 drink per week on average or $>2$ drinks on any one occasion, although most control subjects had no PAE during any trimester of pregnancy. Exposure information was available for 62 of 63 control subjects, and only two reported any prenatal alcohol exposure: one subject was exposed to an average of 1 drink per month throughout pregnancy, and another was exposed to 3 total drinks during the second trimester only (no exposure during the first or third trimesters). Alcohol-exposed subjects had heavy PAE, defined as exposure to $>4$ drinks per occasion at least once per week or $>13$ drinks per week. Subjects without adequate documentation were also classified as PAE if there was both reasonable suspicion of heavy prenatal alcohol exposure or documented maternal history of alcohol abuse, and the physical characteristics of fetal alcohol syndrome. Detailed exposure histories were available for 37 of 70 alcohol-exposed subjects ( 6 from Los Angeles, 1 from San Diego, 30 from Cape Town). Exposure information was provided in two variables: frequency of drinking days and average drinks per occasion. From these variables, we calculated an approximate number of drinks per week, which averaged 15 drinks/week during the first trimester, 13 drinks/week during the second trimester, and 10 drinks/week during the third trimester.

Control subjects were recruited from ongoing studies at each site or specifically for this study via advertisements, word of mouth, or national registers (Mattson et al., 2010; Roussotte et al., 2012). In Los Angeles, alcohol-exposed subjects were recruited primarily through the University of California at Los Angeles (UCLA) Fetal Alcohol and Related Disorders Clinic. Alcohol exposure for these subjects was established through parent/guardian interviews and social, medical, and/or legal records when available. In Cape Town, alcohol-exposed subjects had participated in a previous study on the epidemiology of fetal alcohol spectrum disorders (FASD) among first graders (May et al., 2007), and were recruited through the University of Cape Town from a small city and its surrounding rural areas in the Western Cape Province, a region with one of the highest documented rates of FASD in the world (May et al., 2000, 2007; Viljoen et al., 2005). Exposure status was reconfirmed at the time of brain imaging through maternal interviews and questionnaires. In San Diego, alcohol-exposed participants were recruited through the San Diego State University (SDSU) Center for Behavioral Teratology. Alcohol exposure was determined through parent/legalguardian reports and review of medical, legal and/or social service records when available. Across all sites, participants were excluded for significant head injury with loss of consciousness for $>30 \mathrm{~min}$, significant physical or psychiatric disability that would prevent participation in the imaging and or cognitive assessments, or other known causes of mental deficiency, such as chromosomal abnormalities.

After an explanation of procedures, all subjects provided assent and their parent/guardian provided written informed consent. The institutional review boards at UCLA, SDSU, and the University of Cape Town approved all procedures.

Cognitive assessments and facial evaluations. Participants were assessed with the Wechsler Intelligence Scale for Children, Fourth Edition (WISC-IV), to obtain a Full Scale IQ score (FSIQ); the neurocognitive aspects of the CIFASD project have been described in more detail previously (Mattson et al., 2010). Most participants at each site were also examined by a trained dysmorphologist for the facial features associated with fetal alcohol syndrome (Jones et al., 2006); 65 alcohol-exposed subjects and 56 control subjects received facial evaluations. Palpebral fissure length (PFL) was measured in millimeters using a rigid ruler against the lower eyelid (Hoyme et al., 2005). The philtrum and upper lip were assessed and ranked separately from 1 to 5 depending on the philtrum smoothness and the lip thinness based on the racially appropriate lipphiltrum guide (Astley and Clarren, 2000); we refer to these as philtrum rank and lip rank, respectively. Shorter PFL and higher philtrum and lip ranks indicate greater dysmorphology. Of 37 alcohol-exposed subjects who received the full physical examination, 23 were classified as having fetal alcohol syndrome based on the Hoyme criteria (Hoyme et al., 2005).

Image acquisition. High-resolution T1-weighted MRI data were collected in Los Angeles, California, on a 1.5 T Siemens Sonata (TR, 1900 ms; TE, $4.38 \mathrm{~ms}$; flip angle, $15^{\circ}$; matrix, $256 \times 256 \times 160$; FOV, $256 \times$ $256 \mathrm{~mm}$; total acquisition time, $8 \mathrm{~min} 8 \mathrm{~s}$ ). Data were collected in Cape Town, South Africa, on a 3 T Siemens Allegra (TR, $2200 \mathrm{~ms}$; TE, $5.16 \mathrm{~ms}$; flip angle, $12^{\circ}$; matrix, $256 \times 256 \times 160$; FOV, $256 \times 256 \mathrm{~mm}$; total acquisition time, $7 \mathrm{~min} 4 \mathrm{~s}$ ). Data were collected in San Diego, California, on a 3 T General Electric Signa Excite (TR, 8 ms; TE, 3.0 ms; flip angle, $12^{\circ}$; matrix, $256 \times 256 \times 192$; FOV, $240 \times 240 \mathrm{~mm}$; total acquisition time, $7 \mathrm{~min} 4 \mathrm{~s}$ ). Final voxel sizes were $1 \times 1 \times 1 \mathrm{~mm}$ for Los Angeles and San Diego, and $0.94 \times 0.94 \times 1 \mathrm{~mm}$ for Cape Town.

Image processing. All data were processed through the longitudinal stream in FreeSurfer v5.1, using robust, inverse consistent registration (Reuter et al., 2010) to create an unbiased within-subject template (Reuter and Fischl, 2011). Information from each subject template was used to initialize the longitudinal image processing, increasing repeatability 
Table 1. Demographic information for the alcohol-exposed and control groups

\begin{tabular}{|c|c|c|c|c|}
\hline & Alcohol-exposed & Control & $t$ Statistic for group differences & $\overline{p \text {-value }}$ \\
\hline Age at first scan (years; $n=70,63$ ) & $12.3 \pm 2.6$ & $12.2 \pm 2.8$ & -0.23 & 0.82 \\
\hline Gender $(n=70,63)$ & 44 male, 26 female & 30 male, 33 female & $1869^{a}$ & 0.08 \\
\hline Handedness $(n=70,63)$ & 64 right, 6 left & 60 right, 3 left & $2121^{a}$ & 0.38 \\
\hline $\operatorname{PFL}(\mathrm{cm} ; n=65,56)$ & $2.42 \pm 0.20$ & $2.55 \pm 0.12$ & 4.5 & $<0.001$ \\
\hline PFL percentile $(n=65,56)$ & $20.8 \pm 20$ & $36.5 \pm 15$ & 5.0 & $<0.001$ \\
\hline Lip rank $(n=65,56)$ & $3.6 \pm 0.8$ & $3.0 \pm 0.9$ & -4.0 & $<0.001$ \\
\hline Philtrum rank $(n=65,55)$ & $3.6 \pm 0.7$ & $3.2 \pm 0.7$ & -3.4 & 0.001 \\
\hline WISC-IV FSIQ; $(n=68,58)$ & $66 \pm 28$ & $78 \pm 29$ & 2.3 & 0.02 \\
\hline Alcohol exposure $1^{\text {st }}$ trimester (approximate \# drinks/week; $n=62,37$ ) & $16 \pm 13$ & $0 \pm 0.1$ & -7.3 & $<0.001$ \\
\hline PAE $2^{\text {nd }}$ trimester (\# drinks/week; $n=63,37$ ) & $13 \pm 14$ & $0 \pm 0.1$ & -5.4 & $<0.001$ \\
\hline PAE $3^{\text {rd }}$ trimester (\# drinks/week; $n=63,38$ ) & $10 \pm 12$ & $0 \pm 0.1$ & -5.2 & $<0.001$ \\
\hline
\end{tabular}

The alcohol-exposed group consisted of 70 subjects. The control group consisted of 63 subjects. Because not all subjects had valid measurements for each variable, actual subject numbers are given in left column for each variable $\left(n_{\text {alcohol-exposed }}, n_{\text {control }}\right)$.

${ }^{a}$ For gender and handedness, the Mann-Whitney $U$ test statistic was used.

and statistical power. Using these processing techniques, we obtained 34 cortical volumes in each hemisphere, as well as the volumes of total cortical gray and white matter and supratentorial volume.

Reliability analysis. To ensure reliability of imaging data across the three sites, one subject (female, aged 41-45 years during the scanning period) was scanned several times at each site (3 times in Los Angeles, twice in San Diego, 4 times in Cape Town) across a period of 4 years. Data from this subject was processed in the same manner described above to determine cortical volume in 68 brain regions. Intraclass correlation coefficients (2-way mixed analysis for consistency) from data within each site were 0.997-0.999, and the intraclass correlation coefficient of the average data from each site was 0.994 , indicating excellent agreement both within and across sites. To compensate for any absolute differences in volume, site was included as a random variable in all analyses.

Statistical analysis. Statistical analysis was performed in SPSS v19 (IBM) and run in two stages: (1) a preliminary analysis for age-related volume changes and (2) a subsequent analysis for age-group interactions. The preliminary analysis tested linear and quadratic age changes within each cortical region using mixed models with site and subject as random variables; alcohol-exposed and control groups were tested separately for these age-related changes. False discovery rate correction was used over the linear and quadratic age terms for both groups in all regions analyzed ( 272 tests); $q<0.05$ was considered significant. Any structures with significant linear and/or quadratic age-related changes in either group were retained for further analysis. Global measures (total white and gray matter, supratentorial volume) were analyzed for interactions regardless of whether they had age-related changes in either group.

Subsequent analysis for different developmental trajectories tested each region for significant age-group and age-squared-group interactions using a similar mixed-model approach with site and individual as random variables, but with both groups combined. Age-group interactions indicate significantly different linear trends between groups, while age-squared-group interactions indicate significantly different quadratic components to the trajectory. For structures included in this subsequent analysis, only linear age-group interactions were tested if the quadratic age-related change term did not meet a significance of $p<0.05$ in either group. At this stage, significance was set to $p<0.05$ for the interaction terms, given the previous stringent correction.

Facial measures (PFL, lip rank, philtrum rank), WISC-IV FSIQ scores, and number of drinks per week in each trimester were tested to determine whether they were significant predictors of cortical volume change (annualized rate of change between scans) for the regions that had significant age changes in the initial analyses. Correlations between facial/ cognitive measures and development were examined within each group separately, only in regions for which that group had a significant $(q<$ 0.05 ) age or age-squared effect in the first stage of analysis. In all cases, site was used as a random variable, and age was included as a covariate.

Subjects were grouped according to the average number of drinks per week during each trimester: groups were $0,1-10,11-20,21-30$, or $31+$ drinks per week. An ANOVA controlling for age at first scan was con- ducted to test for group differences of annualized rate of change of cortical volume in each region with significant age-related changes.

An additional analysis was conducted to test for gender differences among regions with significant age-related changes. Gender was included in the model, along with the relevant interaction terms with group, age, and age-squared.

\section{Results}

Cognitive assessments, facial evaluations, and exposure histories Demographics for each group are shown in Table 1. Subjects with PAE had significantly greater facial dysmorphology than controls (shorter PFL, higher lip and philtrum ranks). Lip and philtrum ranks of 4 and 5 indicate the most severe dysmorphology. Among the 55 control subjects with philtrum ranks, 15 (27\%) and $1(2 \%)$ had ranks of 4 and 5, respectively; of the 65 alcohol-exposed subjects, 31 (48\%) and $6(9 \%)$ had ranks of 4 and 5, respectively. For lip rank, $13(21 \%)$ and $2(3 \%)$ control subjects ranked 4 or 5 , respectively, while $26(37 \%)$ and $8(11 \%)$ subjects with PAE ranked 4 and 5, respectively. Two controls (2\%) and 30 exposed subjects $(46 \%)$ had PFL below the 10th percentile. Groups did not differ on age or gender distribution. WISC-IV FSIQ scores were significantly lower in the alcohol-exposed group than in controls, although the mean IQ of control subjects was still considerably below the normal population mean of 100. Among alcohol-exposed individuals, PAE was significantly higher during all three trimesters compared with control subjects, who had very minimal exposure.

\section{Trajectories of overall brain volumes}

Total cortical gray matter volume followed an inverted U-shaped trajectory in both subject groups. However, the quadratic portion was more pronounced in controls, while those with PAE exhibited a more linear decline (Fig. 1). White matter volume linearly increased across the age range in both groups, and the alcoholexposed group had consistently less white matter than controls. In control subjects, total cerebral volume slightly increased and then decreased. Meanwhile in the PAE group, total cerebral volume changed very little. No age-group interaction terms were significant for gray matter, white matter, or total cerebral volume, indicating no significant differences between group trajectories.

\section{Age-related changes within regions}

Of 68 cortical regions, 34 had significant $(q<0.05)$ quadratic age-related changes, and 27 had linear age-related changes (Fig. $2)$. In six regions where the linear term met the stringent significance criterion $(q<0.05)$, the quadratic term remained significant at $p<0.05$. Thus, for 40 regions, both linear and quadratic 

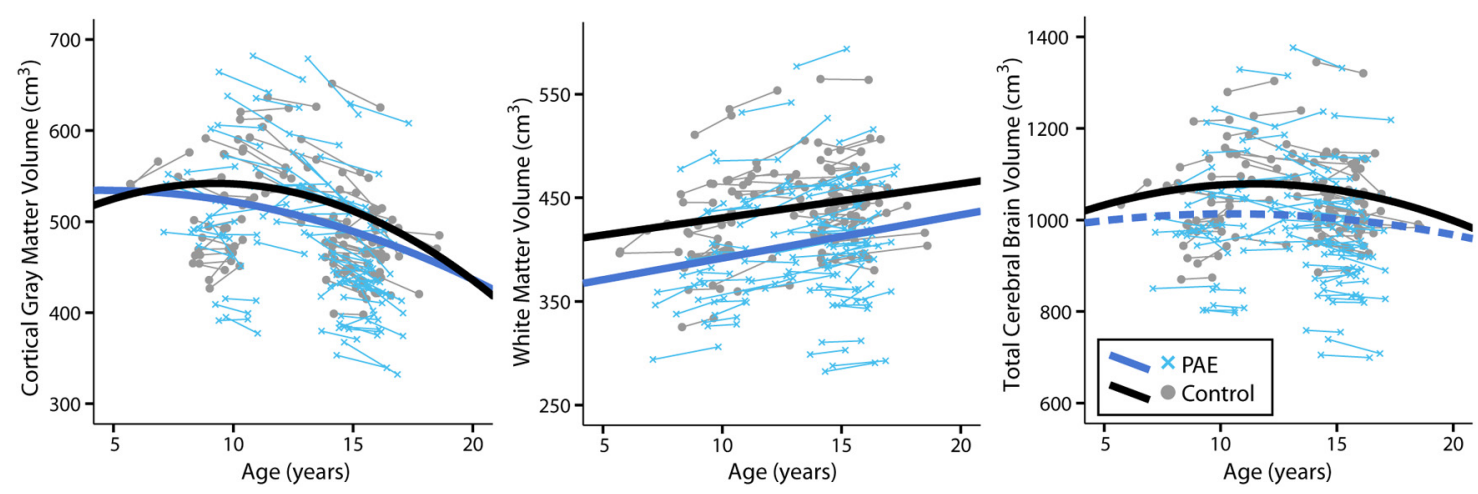

Figure 1. Developmental trajectories for cortical gray matter, white matter, and total cerebral volumes for control subjects (black) and those with PAE (blue). For PAE subjects, the total brain volume trend was not significant (dashed line). Trajectories were not significantly different between groups for any of these three measures.

interaction terms were tested, and for 21 regions, linear (and no quadratic) agegroup interactions were tested to determine whether trajectory differences existed between subjects with and without PAE. In general, control subjects exhibited more quadratic trajectories of volume change than the subjects with PAE.

Differences in developmental trajectories Of the regions examined for group differences in developmental trajectories, six had significant age-squared-group interactions $(p<0.05)$, indicating significantly different patterns of maturation. No regions had significant linear agegroup interactions. The six regions with significant trajectory differences were primarily located in posterior brain regions, specifically in the inferior and superior parietal regions, as well as the banks of the superior temporal sulcus and the postcentral gyrus (Fig. 3). In all of these regions, control subjects had more curvature to their development trajectories than subjects with PAE, who exhibited less overall change in cortical volume.

\section{Gender effects}

Of the regions with significant age effects, eight had significant age-gender interaction effects. The right and left medial orbitofrontal, the right lateralorbitofrontal, and the right middle temporal regions had significant linear age-gender interactions. In all of these areas, females exhibited faster volume declines than males. In the right precentral region, there was a significant age-squared-gender interaction such that females showed a more linear volume decline across the age range, while males had a more curved quadratic trajectory with increases followed by decreases. Three left-hemisphere areas had significant age-squared-group-gender interactions: the precentral, superior parietal, and supramarginal regions. In all three of these regions, male control subjects had more quadratic trajectories of volume change than the other three groups, indicating a longer period of volume increases. The left superior parietal area was the only one with gender effects that also had significant age-group interactions; however, the gender inter- actions here did not remain significant when all terms were included in the overall model, while the quadratic age-group interaction remained significant.

\section{Correlations with facial morphology}

Correlations between development rates and facial measures (PFL, lip rank, philtrum rank) occurred in all brain lobes, but were most prominent in frontal cortices (Fig. 4). Subjects with PAE demonstrated 32 significant correlations between development rates and facial dysmorphology (in 22 distinct regions), most of which were in frontal lobes. Control subjects had 14 significant correlations (in 10 distinct regions), which were focused in posterior brain areas. The vast majority of correlations with facial measures were such that smaller decreases of brain volume were associated with worse facial morphology. The only 

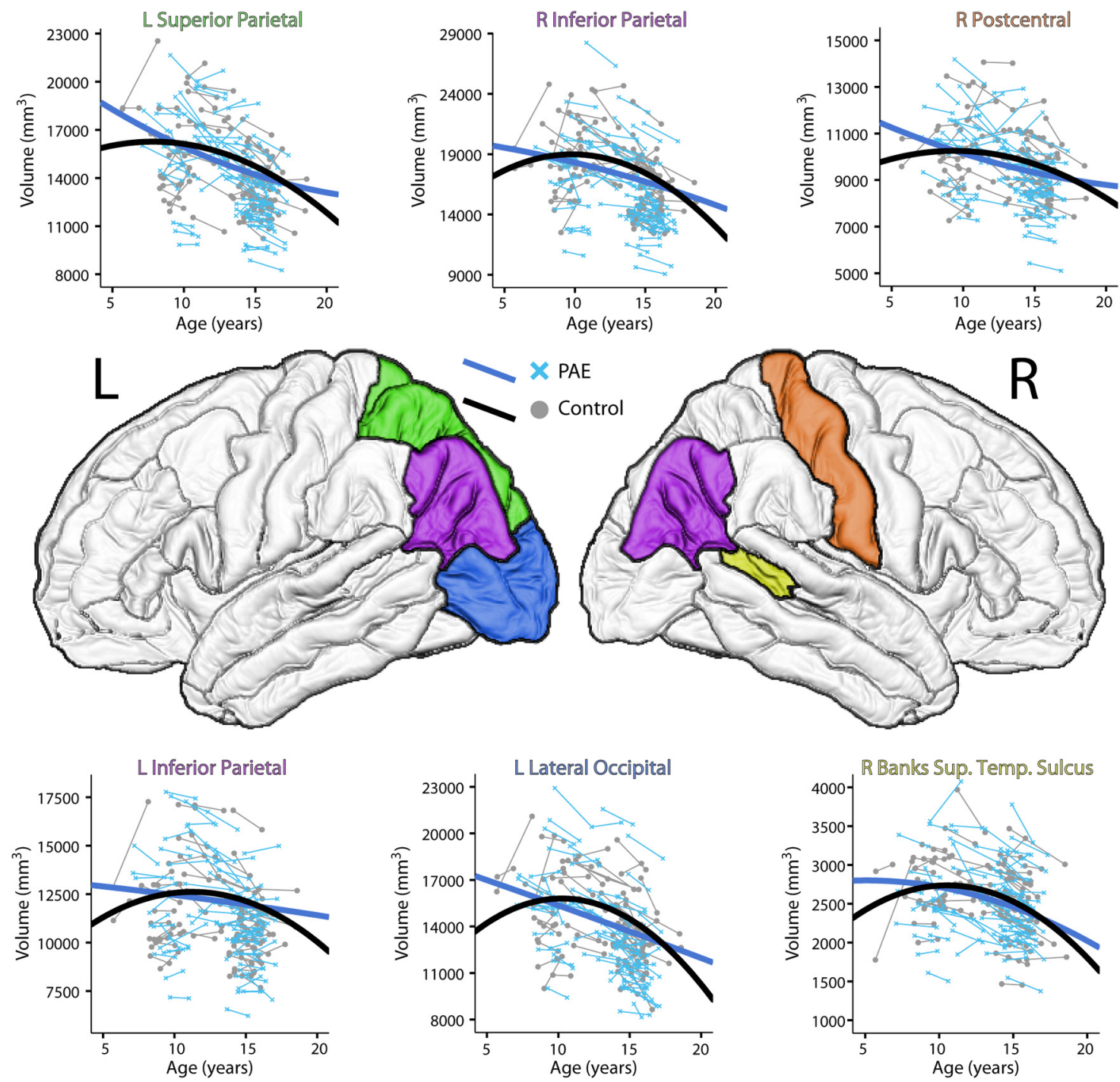

Figure 3. Brain regions with significantly different development trajectories between subjects with PAE and controls are shown in various colors on the brain surface, with accompanying volume-age plots. In general, the PAE trajectories (blue) showed more linear trends with smaller magnitudes of change (both increasing and decreasing volumes) than controls (black), suggesting reduced brain plasticity.

exceptions to this pattern were in the frontal pole, where controls demonstrated a positive correlation with PFL (right side), and subjects with PAE had negative correlations with lip rank (left side); in both cases, these correlations indicate larger decreases of brain volume are associated with worse facial morphology.

Correlations with cognition and quantities of alcohol exposure Correlations between WISC-IV FSIQ and annualized cortical volume changes were observed in several posterior brain regions for controls, and in two frontal regions in subjects with PAE (Fig. $5)$. Most correlations were positive, indicating a relationship between higher IQ and larger volume decreases. The left frontal pole had a positive relationship between IQ and rates of change in the PAE group, such that lower IQ was associated with larger volume decreases. Rates of cortical change were associated with quantities of alcohol exposure in temporal and cingulate cortices (Fig. 5). The temporal lobe regions (right transverse temporal, left parahippomcampal) had negative correlations, indicating that greater volumes of prenatal alcohol exposure were associated with larger decreases of volume between scans. In the left posterior cingulate, correlations were positive, indicating that in- creased alcohol exposure was associated with smaller volume decreases between scans. Because detailed exposure information was primarily available from the subjects in South Africa $(n=32$ from Cape Town; $n=6$ from California), the relationships observed between exposure amounts and brain development may not be representative of this entire sample.

\section{Discussion}

This longitudinal study used structural MRI to demonstrate altered trajectories of brain maturation in children and youth with heavy in utero alcohol exposure. While unexposed control children show a plastic cortex with a prolonged pattern of cortical volume increases followed by equally vigorous volume decreases, individuals with PAE showed only volume loss in most cortical areas; trajectory differences were significant in posterior cortices bilaterally. In both groups, higher overall intellectual functioning and more normal facial morphology were associated with greater volume reductions; these correlations were focused in posterior brain regions for control subjects, but spread throughout the brain in PAE subjects. Finally, within the PAE group, volume 

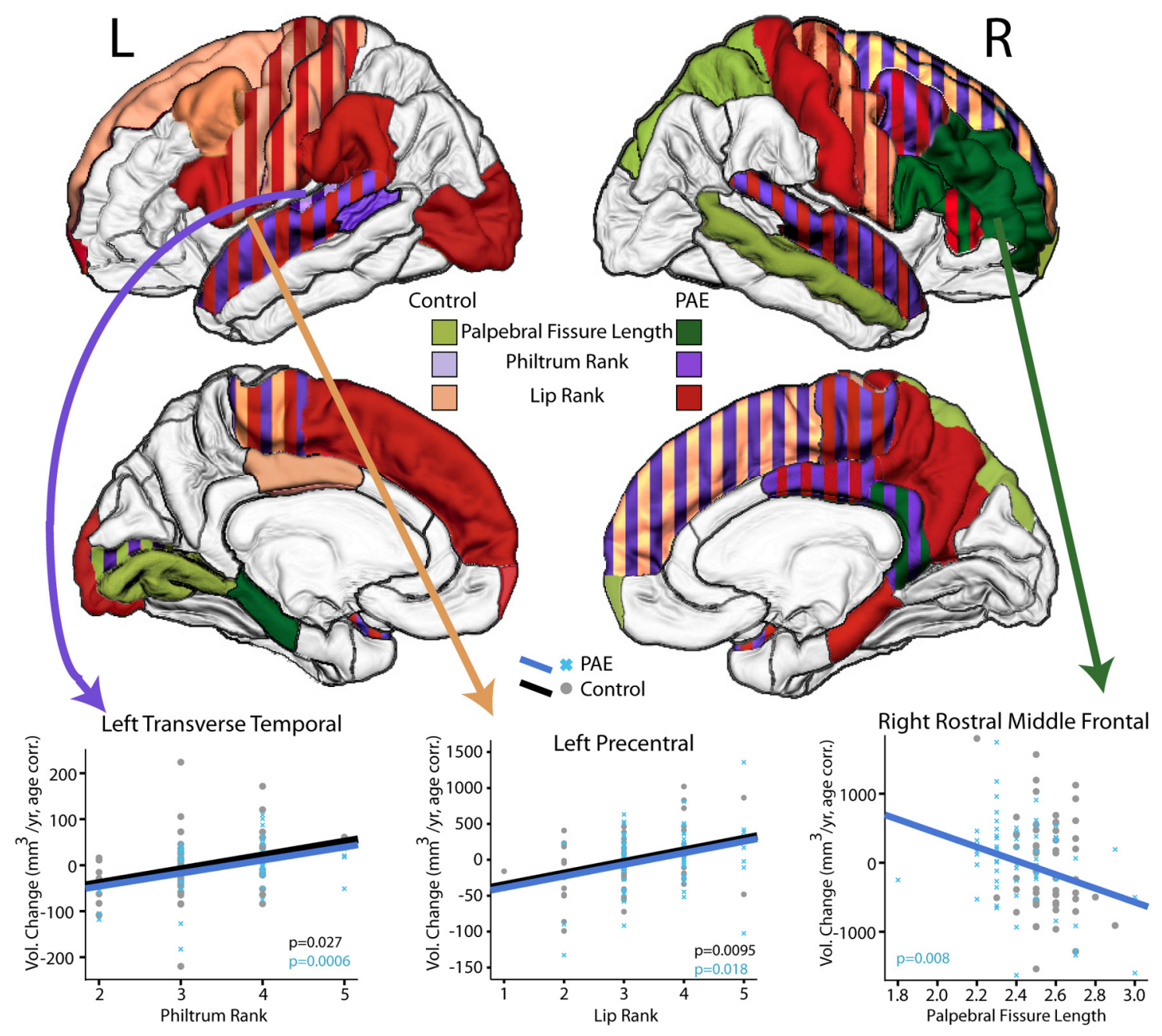

Figure 4. Correlations between cortical volume development and facial dysmorphology (PFL, lip rank, philtrum rank) were significant in many brain regions, indicated above with color. Plots from three representative regions are shown to illustrate relationships. In all cases except the frontal pole, worse facial dysmorphology (higher lip or philtrum rank or shorter PFL) was related to more gradual brain development.

changes in the temporal and cingulate cortices were negatively and positively associated with the amount of alcohol exposure in utero, indicating that increased alcohol exposure was associated with greater and lesser volume reductions between scans, respectively.

The findings presented here support the notion of trade-offs between plasticity and efficiency, such that children who have a more prolonged period of brain growth and plasticity (marked by cortical volume increases) followed by more synaptic pruning and myelination (which would appear as volume reductions over time on MRI) within posterior cortices process information more efficiently. In contrast, children with PAE exhibit less plasticity and may prematurely prune connections that could ultimately have been useful in developing more efficient functioning.

The differences in maturational trajectories observed may be due to PAE or may be related to prolonged dysfunctional experiences throughout childhood and adolescence. Children with PAE experience more cognitive, behavioral, and social difficulties than their unexposed peers (Mattson et al., 2011; Riley et al., 2011). Experiences within one's environment help build brain connections, and these experiences are highly likely to differ in children with neurodevelopmental disorders because of the deficits such children have. It has long been known from animal studies that environmental enrichment can affect the architecture of the brain, and that there are critical periods during which the brain is primed to adapt to certain types of external sensory input (for review, see Baroncelli et al., 2010). Even in adults, structural brain changes have been observed with MRI after learning new skills (for review, see Fields, 2011). The same could be happening in children with PAE and other neurodevelopmental disorders where the wiring of the brain throughout development is happening either in the absence of functional behaviors within the environment or in the presence of behaviors that are dysfunctional. Future studies with more detailed histories may be able to examine the separate effects of prenatal alcohol exposure and childhood environments, providing valuable insight into the factors contributing to altered development trajectories.

In this report, general intellectual functioning predicted development rates in posterior cortices in control subjects and more widespread areas in individuals with PAE. Relationships between gray matter development trajectories and IQ have been shown previously, with children of superior intelligence demonstrating later peak cortical thickness than children with average or high intelligence (Shaw et al., 2006). The results here suggest a similar relationship in posterior cortices of children of low-toaverage IQ but with no prenatal exposure and in the rostral mid- 

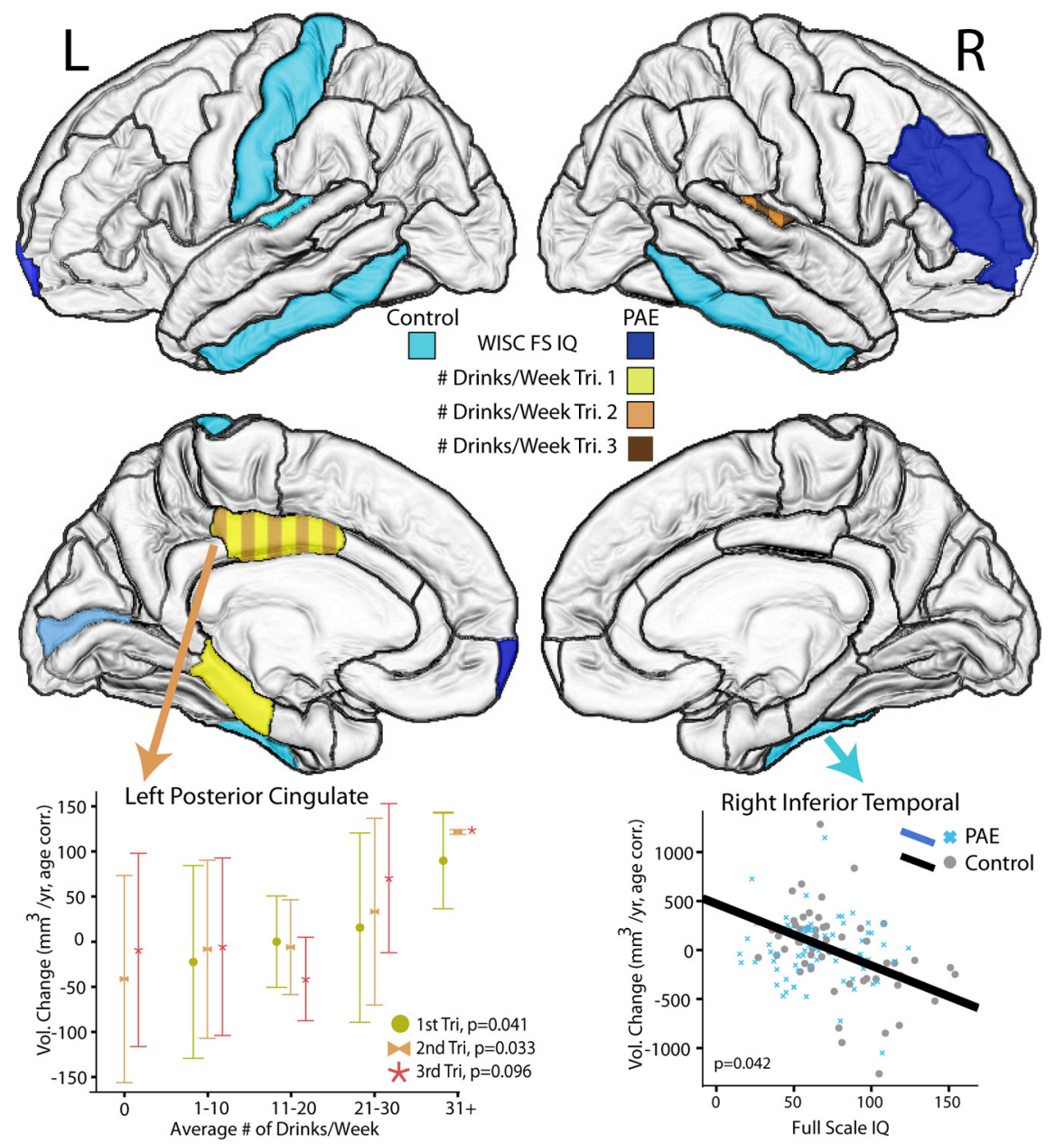

Figure 5. Correlations between annualized rates of cortical change and $I Q$ or PAE for each trimester were significant in several regions, primarily in posterior and temporal cortices. In most cases, higher IQ and smaller amounts of PAE were associated with larger volume decreases between scans. Data from selected regions are shown as examples.

dle frontal region in subjects with PAE; that is, subjects with lower IQ values had less overall volume change than those with higher IQ. However, in the frontal pole region, subjects with PAE demonstrated the opposite effect: higher IQ was associated with less overall volume change. Although IQ values have been generally assumed to be stable over the lifetime, new evidence suggests that they may change during adolescence (Ramsden et al., 2011). Specifically, verbal IQ increases over time were associated with increased gray matter density in the motor speech area, an area just anterior to the postcentral region where we observed correlations between FSIQ and gray matter volume loss in control subjects. The results presented here and previous findings regarding longitudinal maturational trajectories and their relationships with intellectual functioning highlight the notion that ongoing cognitive and behavioral deficits within the developmental environment (e.g., experience-dependent plasticity) may compound the impact of damage (e.g., heavy PAE) that caused the dysfunction in the first place. Future studies including children with other neurodevelopmental disorders may elucidate the multiple confounding influences on on-going dysfunction within the developmental environment, which may be independent from the etiology of this disorder.

Facial morphology was associated with development rates in both groups. Several studies have demonstrated correlations between brain structure and facial dysmorphology at a single time point in subjects with PAE (Astley et al., 2009; Roussotte et al., 2012; Yang et al., 2012b), including one study that related inferior temporal cortical thickness to PFL in an overlapping group of subjects, cross-sectionally at time 1 (Yang et al., 2012a). Here, correlations with facial measures indicated that larger volume decreases were associated with more normal facial morphology. The only exception was the frontal pole, where smaller volume decreases were observed in subjects with the most normal facial morphology (PFL and lip rank). Correlations between facial characteristics and brain structure are not entirely surprising in control subjects, as the brain and the face develop at similar times in utero, and these relationships are not necessarily indicative of cognitive or behavioral dysfunction. The brain-face relationships were much more widespread in the alcohol-exposed individuals, for whom facial dysmorphology predicted brain changes in multiple brain areas. Except for the frontal pole, worse facial dysmorphology (higher lip or philtrum ranks, shorter PFL) was associated with less change between scans (smaller volume decreases) in subjects with PAE.

It is possible that the timing and quantity of PAE at any given time point, particularly during the early phases of pregnancy, when the face is forming, affect both the brain and the face more negatively than exposure during later stages of pregnancy, when the face is already more fully formed (O'LearyMoore et al., 2011). While it is difficult to quantify the timing and severity of exposure in retrospective human studies (May et al., 2008), it is well known from animal studies and limited human research that quantity, frequency, and timing of alcohol exposure influence dysmorphology of the brain and the face (Sulik, 2005), and correlate with intellectual and behavioral functioning (Viljoen et al., 2005; May et al., 2008). In this sample, greater alcohol exposure during pregnancy was associated with smaller volume decreases in the cingulate cortex and greater volume decreases in temporal areas in children and youth with PAE.

Most correlations with IQ, facial measures, and alcohol exposure indicated that more normal features are associated with greater decreases of volume between scans. Parietal and occipital brain cortices are known to develop most rapidly between childhood and adolescence, whereas frontal and temporal cortices continue to mature between adolescence and adulthood (Sowell et al., 1999; Gogtay et al., 2004). Thus, it seems reasonable to speculate that greater volume loss in the posterior cortices, which occurs prominently between childhood and adolescence, would be a "good thing," whereas greater volume loss in some frontal and temporal cortices could be a "bad thing" in the age range studied, as it would normally occur later. These patterns are also true of the present data, which show that, in controls, the frontal and temporal regions, compared with parietal and occipital cortices, had more prolonged maturation. Furthermore, although trajectories were not significantly different between groups, 
alcohol-exposed subjects demonstrated steeper volume drops during adolescence than controls in frontal and temporal cortices, whereas they tended to have less overall change in parietal and occipital areas than control subjects.

Though the mechanisms remain somewhat unclear, the parietal cortices have been consistently implicated as abnormal in children and adolescents prenatally exposed to alcohol, demonstrating reduced cortical volume (Archibald et al., 2001; Chen et al., 2012), increased gray matter density (Sowell et al., 2001), and altered shape (Sowell et al., 2002), in addition to white matter abnormalities in pathways connecting to parietal cortices (Lebel et al., 2008; Sowell et al., 2008b). Cortical thickness is altered in parietal areas, though some studies report thicker cortices in children and youth with PAE (Sowell et al., 2008a; Yang et al., 2012a), and one study found thinner cortices in alcohol-exposed subjects (Zhou et al., 2011). The differing cortical volume trajectories observed here may help explain the apparently conflicting findings in previous studies, as the differences between subjects with PAE and unexposed individuals change over time.

In conclusion, we observed significantly altered development trajectories in children and youth with heavy PAE. These individuals demonstrated less volume change over time than their unexposed peers, suggesting reduced brain plasticity. Furthermore, this reduced plasticity was related to lower IQ and more severe facial dysmorphology. These long-term effects may be due specifically to drinking during pregnancy, but are likely also influenced by dysfunctional childhood experiences. These observations indicate that brain abnormalities in children and youth with PAE are not static, and have important implications for early treatments and interventions within this disorder (Adnams et al., 2007) that may be able to "correct" the developmental trajectories. Finally, these implications may not be limited specifically to in utero alcohol exposure, but are likely also relevant to other neurodevelopmental disorders.

\section{References}

Adnams CM, Sorour P, Kalberg WO, Kodituwakku P, Perold MD, Kotze A, September S, Castle B, Gossage J, May PA (2007) Language and literacy outcomes from a pilot intervention study for children with fetal alcohol spectrum disorders in South Africa. Alcohol 41:403-414.

Archibald SL, Fennema-Notestine C, Gamst A, Riley EP, Mattson SN, Jernigan TL (2001) Brain dysmorphology in individuals with severe prenatal alcohol exposure. Dev Med Child Neurol 43:148-154.

Astley SJ, Clarren SK (2000) Diagnosing the full spectrum of fetal alcoholexposed individuals: introducing the 4-digit diagnostic code. Alcohol Alcohol 35:400-410.

Astley SJ, Aylward EH, Olson HC, Kerns K, Brooks A, Coggins TE, Davies J, Dorn S, Gendler B, Jirikowic T, Kraegel P, Maravilla K, Richards T (2009) Magnetic resonance imaging outcomes from a comprehensive magnetic resonance study of children with fetal alcohol spectrum disorders. Alcohol Clin Exp Res 33:1671-1689.

Baroncelli L, Braschi C, Spolidoro M, Begenisic T, Sale A, Maffei L (2010) Nurturing brain plasticity: impact of environmental enrichment. Cell Death Differ 17:1092-1103.

Chen X, Coles CD, Lynch ME, Hu X (2012) Understanding specific effects of prenatal alcohol exposure on brain structure in young adults. Hum Brain Mapp 33:1663-1676.

Fields RD (2011) Imaging learning: the search for a memory trace. Neuroscientist 17:185-196.

Giedd JN, Rapoport JL (2010) Structural MRI of pediatric brain development: what have we learned and where are we going? Neuron 67:728-734.

Giedd JN, Lenroot RK, Shaw P, Lalonde F, Celano M, White S, Tossell J, Addington A, Gogtay N (2008) Trajectories of anatomic brain development as a phenotype. Novartis Found Symp 289:101-112; discussion 112-118, 193-195.

Gogtay N, Giedd JN, Lusk L, Hayashi KM, Greenstein D, Vaituzis AC, Nugent TF 3rd, Herman DH, Clasen LS, Toga AW, Rapoport JL, Thompson PM
(2004) Dynamic mapping of human cortical development during childhood through early adulthood. Proc Natl Acad Sci U S A 101:8174-8179.

Hoyme HE, May PA, Kalberg WO, Kodituwakku P, Gossage JP, Trujillo PM, Buckley DG, Miller JH, Aragon AS, Khaole N, Viljoen DL, Jones KL, Robinson LK (2005) A practical clinical approach to diagnosis of fetal alcohol spectrum disorders: clarification of the 1996 institute of medicine criteria. Pediatrics 115:39-47.

Huttenlocher PR (1979) Synaptic density in human frontal cortex-developmental changes and effects of aging. Brain Res 163:195-205.

Huttenlocher PR, de Courten C (1987) The development of synapses in striate cortex of man. Hum Neurobiol 6:1-9.

Jones KL, Robinson LK, Bakhireva LN, Marintcheva G, Storojev V, Strahova A, Sergeevskaya S, Budantseva S, Mattson SN, Riley EP, Chambers CD (2006) Accuracy of the diagnosis of physical features of fetal alcohol syndrome by pediatricians after specialized training. Pediatrics 118 : e1734-e1738.

Lebel C, Rasmussen C, Wyper K, Walker L, Andrew G, Yager J, Beaulieu C (2008) Brain diffusion abnormalities in children with fetal alcohol spectrum disorder. Alcohol Clin Exp Res 32:1732-1740.

Mattson SN, Foroud T, Sowell ER, Jones KL, Coles CD, Fagerlund A, AuttiRämö I, May PA, Adnams CM, Konovalova V, Wetherill L, Arenson AD, Barnett WK, Riley EP (2010) Collaborative initiative on fetal alcohol spectrum disorders: methodology of clinical projects. Alcohol 44:635641.

Mattson SN, Crocker N, Nguyen TT (2011) Fetal alcohol spectrum disorders: neuropsychological and behavioral features. Neuropsychol Rev 21: 81-101.

May PA, Brooke L, Gossage JP, Croxford J, Adnams C, Jones KL, Robinson L, Viljoen D (2000) Epidemiology of fetal alcohol syndrome in a South African community in the Western Cape Province. Am J Public Health 90:1905-1912.

May PA, Gossage JP, Marais AS, Adnams CM, Hoyme HE, Jones KL, Robinson LK, Khaole NC, Snell C, Kalberg WO, Hendricks L, Brooke L, Stellavato C, Viljoen DL (2007) The epidemiology of fetal alcohol syndrome and partial FAS in a South African community. Drug Alcohol Depend 88:259-271.

May PA, Gossage JP, Marais AS, Hendricks LS, Snell CL, Tabachnick BG, Stellavato C, Buckley DG, Brooke LE, Viljoen DL (2008) Maternal risk factors for fetal alcohol syndrome and partial fetal alcohol syndrome in South Africa: a third study. Alcohol Clin Exp Res 32:738-753.

Nardelli A, Lebel C, Rasmussen C, Andrew G, Beaulieu C (2011) Extensive deep gray matter volume reductions in children and adolescents with fetal alcohol spectrum disorders. Alcohol Clin Exp Res 35:1404-1417.

O’Leary-Moore SK, Parnell SE, Lipinski RJ, Sulik KK (2011) Magnetic resonance-based imaging in animal models of fetal alcohol spectrum disorder. Neuropsychol Rev 21:167-185.

Ramsden S, Richardson FM, Josse G, Thomas MS, Ellis C, Shakeshaft C, Seghier ML, Price CJ (2011) Verbal and non-verbal intelligence changes in the teenage brain. Nature 479:113-116.

Reuter M, Fischl B (2011) Avoiding asymmetry-induced bias in longitudinal image processing. Neuroimage 57:19-21.

Reuter M, Rosas HD, Fischl B (2010) Highly accurate inverse consistent registration: a robust approach. Neuroimage 53:1181-1196.

Riley EP, Infante MA, Warren KR (2011) Fetal alcohol spectrum disorders: an overview. Neuropsychol Rev 21:73-80.

Roussotte FF, Sulik KK, Mattson SN, Riley EP, Jones KL, Adnams CM, May PA, O’Connor MJ, Narr KL, Sowell ER (2012) Regional brain volume reductions relate to facial dysmorphology and neurocognitive function in fetal alcohol spectrum disorders. Hum Brain Mapp 33:920-937.

Shaw P, Greenstein D, Lerch J, Clasen L, Lenroot R, Gogtay N, Evans A, Rapoport J, Giedd J (2006) Intellectual ability and cortical development in children and adolescents. Nature 440:676-679.

Shaw P, Eckstrand K, Sharp W, Blumenthal J, Lerch JP, Greenstein D, Clasen L, Evans A, Giedd J, Rapoport JL (2007) Attention-deficit/hyperactivity disorder is characterized by a delay in cortical maturation. Proc Natl Acad Sci U S A 104:19649-19654.

Sowell ER, Thompson PM, Holmes CJ, Jernigan TL, Toga AW (1999) In vivo evidence for post-adolescent brain maturation in frontal and striatal regions. Nat Neurosci 2:859-861.

Sowell ER, Thompson PM, Mattson SN, Tessner KD, Jernigan TL, Riley EP, Toga AW (2001) Voxel-based morphometric analyses of the brain in 
children and adolescents prenatally exposed to alcohol. Neuroreport 12:515-523.

Sowell ER, Thompson PM, Peterson BS, Mattson SN, Welcome SE, Henkenius AL, Riley EP, Jernigan TL, Toga AW (2002) Mapping cortical gray matter asymmetry patterns in adolescents with heavy prenatal alcohol exposure. Neuroimage 17:1807-1819.

Sowell ER, Mattson SN, Kan E, Thompson PM, Riley EP, Toga AW (2008a) Abnormal cortical thickness and brain-behavior correlation patterns in individuals with heavy prenatal alcohol exposure. Cereb Cortex 18:136-144.

Sowell ER, Johnson A, Kan E, Lu LH, Van Horn JD, Toga AW, O'Connor MJ, Bookheimer SY (2008b) Mapping white matter integrity and neurobehavioral correlates in children with fetal alcohol spectrum disorders. J Neurosci 28:1313-1319.

Sulik KK (2005) Genesis of alcohol-induced craniofacial dysmorphism. Exp Biol Med (Maywood) 230:366-375.

Thompson PM, Vidal C, Giedd JN, Gochman P, Blumenthal J, Nicolson R, Toga AW, Rapoport JL (2001) Mapping adolescent brain change reveals dynamic wave of accelerated gray matter loss in very early-onset schizophrenia. Proc Natl Acad Sci U S A 98:11650-11655.

Vidal CN, Rapoport JL, Hayashi KM, Geaga JA, Sui Y, McLemore LE, Alaghband Y, Giedd JN, Gochman P, Blumenthal J, Gogtay N, Nicolson R, Toga AW, Thompson PM (2006) Dynamically spreading frontal and cingu- late deficits mapped in adolescents with schizophrenia. Arch Gen Psychiatry 63:25-34.

Viljoen DL, Gossage JP, Brooke L, Adnams CM, Jones KL, Robinson LK, Hoyme HE, Snell C, Khaole NC, Kodituwakku P, Asante KO, Findlay R, Quinton B, Marais AS, Kalberg WO, May PA (2005) Fetal alcohol syndrome epidemiology in a South African community: a second study of a very high prevalence area. J Stud Alcohol 66:593-604.

Yakovlev PI, Lecours A-R (1967) The myelogenetic cycles of regional maturation of the brain. In: Regional development of the brain early in life (Minkowski A, ed), pp 3-70. Boston: Blackwell Scientific.

Yang Y, Roussotte F, Kan E, Sulik KK, Mattson SN, Riley EP, Jones KL, Adnams CM, May PA, O’Connor MJ, Narr KL, Sowell ER (2012a) Abnormal cortical thickness alterations in fetal alcohol spectrum disorders and their relationships with facial dysmorphology. Cereb Cortex 22:1170-1179.

Yang Y, Phillips OR, Kan E, Sulik KK, Mattson SN, Riley EP, Jones KL, Adnams CM, May PA, O'Connor MJ, Narr KL, Sowell ER (2012b) Callosal thickness reductions relate to facial dysmorphology in fetal alcohol spectrum disorders. Alcohol Clin Exp Res. 36:798-806.

Zhou D, Lebel C, Lepage C, Rasmussen C, Evans A, Wyper K, Pei J, Andrew G, Massey A, Massey D, Beaulieu C (2011) Developmental cortical thinning in fetal alcohol spectrum disorders. Neuroimage 58:16-25. 